\title{
Effect of SARS-CoV-2 pandemic on breast cancer stage at diagnosis
}

\author{
๑Murat Bulut Özkan, ๑Veysel Barış Turhan, @Murat Baki Yıldırım, @Ramazan Topçu \\ Hitit University, Erol Olçok Training and Research Hospital, General Surgery Clinic, Çorum, Turkey
}

Cite this article as: Özkan MB, Turhan VB, Yıldırım MB, Topçu R. Effect of SARS-CoV-2 pandemic on breast cancer stage at diagnosis. J Health Sci Med 2022; 5(1): 62-66.

\begin{abstract}
Objective: We aimed to research the effects of the COVID-19 pandemic on breast cancer stages at the time of diagnosis.

Material and Method: The data of female patients over 18 who underwent breast surgery and sentinel lymph node sampling for malignancy between 01.06.2019 and 31.11.2019 with between 01.06.2020 and 31.11.2020 were analyzed. Patients were divided into two groups as before and during the pandemic.

Results: Data of 55 patients in total were reached, of which 31 were diagnosed before the pandemic and 24 after the pandemic. There is no significant difference between the two groups in terms of age. Average tumor size is $3.42 \pm 2.00 \mathrm{~cm}$, and 18 patient (32.7\%) has positive sentinel lymph node biopsy (SLNB). In before pandemic group (Group 1) SLNB positivity rate is only $25.8 \%$ $(\mathrm{n}=8)$ but in during pandemic group (Group 2$)$ this rate reaches $41.7 \%(\mathrm{n}=10)$, but this is not statistically significant $(\mathrm{p}=0.214)$. While the tumor size of the patients in Group 1 was $3.35 \pm 2.25 \mathrm{~cm}$, it was $3.51 \pm 1.67 \mathrm{~cm}$ in Group 2. Still, no statistically significant difference was observed $(\mathrm{p}=0.141)$.

Conclusion: As a result of our study, although statistically insignificant, an increase in tumor sizes and positive lymph node numbers was detected. We predict that statistically significant results will be obtained in studies with a larger number of cases.
\end{abstract}

Keywords: COVID-19, SARS-CoV2, breast cancer, SLNB, prognosis

\section{INTRODUCTION}

The SARS-CoV-2 disease was first reported to the World Health Organization (WHO) on 31 December 2019 in Wuhan City, Hubei Province, China (1). WHO defined this disease as COVID-19 on 11 February 2020 (2). During the pandemic, especially at the beginning of the quarantine, there was a significant slowdown in clinics that could make the oncological diagnoses and organize treatments, and only emergency medical services were resumed (3). Lai et al. (4) found a $60 \%$ reduction in patients receiving chemotherapy and a $76 \%$ reduction in referrals for early diagnosis. It has been shown that cancer-related deaths increase in this process (5). Thus, cancer-related deaths may have risen since cancer patients caught COVID-19, as well as the disruption of treatment services and delays in diagnosis during the pandemic. While emerging vaccines show great promise, not everyone's access to vaccines and the emergence of new variant viruses indicate that it will take some time before health services, including cancer services, return to pre-pandemic capacity and cancer prevention services.
Also, it has been shown that patients with co-morbidities are more prone to COVID-19, and in this population, this infection is more hazardous $(6,7)$.

Breast cancer is the most commonly diagnosed cancer worldwide. 2,3 million new cases are diagnosed annually (6). If breast cancer is caught at an early stage, a significant survival advantage is provided. While the one-year survival rate of breast cancer diagnosed in Stage 1 is $100 \%$ in recent studies, and this rate drops to $66 \%$ for Stage 4. For this reason, screening tests are carried out in many countries. Screening programs reduce breast cancer mortality by $20 \%$ (7). In addition, early diagnosis and treatment provide good aesthetic results, reduce the need for adjuvant therapy, provide an early return to work, and improve the quality of life.

During the pandemic, there has been a prominent decrease in hospital admissions and delays in diagnosis and treatment. In addition, some countries had to postpone outpatient services and screening programs due to the overload in their health systems during the COVID-19 
pandemic. Besides, national screening programs in many countries, including Turkey, have been completely suspended for 1-6 months (8). Even in countries where screening services are being continued, attendance has been reported to have decreased by almost 50\% (9).

Our study aims to evaluate the effect of suspension of screening and outpatient services on the stage of breast cancer at diagnosis during the COVID-19 pandemic.

\section{MATERIAL AND METHOD}

The study was carried out with the permission of Hitit University Non-Interventional Research Ethics Committee (Date: 30.04.2021, Decision No: 2021-66) for our retrospective study. Our study was carried out under the principles of the Declaration of Helsinki and an informed consent form was signed by all patients. Between these dates, demographic data, sentinel lymph node positivity status, number of lymph nodes removed, tumor sizes, and whether they received neoadjuvant treatment were retrospectively questioned from patient files and the hospital data systems.

\section{Study Group}

This study was conducted on female patients over 18 years of age who underwent breast surgery and sentinel lymph node biopsy (SLNB) due to malignancy in the General Surgery Clinic of Hitit University Erol Olçok Training and Research Hospital between 01.06.2019 and 31.11.2019 with 01.06.2020 and 31.11.2020.

While determining the dates of patient selection, attention was paid to the absence of April and May, when quarantine was most intense, and elective surgeries were suspended, and patients diagnosed within six months starting from June were included in the study. The dates for screening the other group of patients (Group 1 or before the pandemic group) were determined considering the projection of this time interval to the pre-pandemic period. Fifty-five patients were found to be eligible for the study.

Exclusion criteria were defined as patients under 18 years of age, non-operated breast cancer patients, patients whose final pathology report was not reported as breast cancer, patients whose data could not be accessed, and patients who did not want to be included in the study. A total of 101 patients were examined, and 55 patients who met the criteria were included in the study.

\section{Study Protocol and Definitions}

Preoperative diagnosis was obtained by core needle biopsy or stereotactic biopsy. In addition, data on previous administration of neoadjuvant chemotherapy were collected from clinical notes. The surgical procedure was performed as breast surgery (breast-conserving surgery or mastectomy) and SLNB in all patients.
Breast surgery includes breast-conserving surgery or mastectomy, depending on tumor size, breast size, and patient's choice. Axillary assessment; SLNB was performed in patients without clinical or radiological evidence of pathological lymph nodes. Afterward, axillary lymph node dissection (ALND) was performed in patients with SLNB positivity.

Patients with clinically or radiologically pathological lymph nodes or findings suggestive of locally advanced breast cancer were referred to neoadjuvant therapy.

SLNB was performed by applying a five cc patent blue injection to the periareolar tissue and pectoral fascia. There are studies showing that periareolar injection for SLNB is superior to peritumoral injection due to its simplicity and high success rate in SLN detection (10). For deeply located tumors, $0.5 \mathrm{ml}$ peritumoral injection is recommended (11). Approximately 10 minutes after the application, the lymph nodes stained with an axillary incision were removed and sent for frozen section examination. Data obtained from surgical specimens were included in the study. Tumor maximum diameters were reported as tumor size in centimeters.

Patients who had breast surgery during the pandemic period were evaluated in our study and considered the during pandemic group (Group 2). These patients were compared with patients who had breast surgery in the same period of the previous year, defined as the before the pandemic group (Group 1). These dates were determined to be correlated with the dates when nonemergency surgery cases were started to be cared for at the outpatient level in Turkey.

\section{Statistical Analysis}

All statistical analyzes were performed using IBM SPSS Statistics for Windows software (version 26; IBM Corp., Armonk, N.Y., USA). Categorical variables, sentinel lymph node positivity, and neoadjuvant treatment status were reported as numbers and percentages. The numerical variables, age and tumor size, were reported as mean value \pm standard deviation and median value in parentheses. The number of lymph nodes removed was reported as median value and minimum and maximum values in parentheses. Relationships between variables were investigated with Pearson and Spearman correlation coefficients. The statistical difference of categorical variables between the groups was evaluated by using the Chi-Square test. Data normal distribution in numerical data were evaluated with the Shapiro Wilks test. The Mann-Whitney U test was used in accordance with the data distribution for the number of lymph nodes removed, tumor size, and age comparisons between the two groups. For the statistical significance level, $\mathrm{p}<0.05$ was accepted. 


\section{RESULTS}

When the mean age of 55 patients constituting the whole group was examined, it was found to be $56.29 \pm 12.84$ years. SLNB was positive in 18 (32.7\%) of 55 patients. The mean tumor size removed from the patients was $3.42 \pm 2$ $\mathrm{cm}$ (Table).

To determine the effect of the pandemic on breast cancer stage at the time of diagnosis, the patients were divided into two groups as "before the pandemic" (Group 1) $(\mathrm{n}=31)$ and "during pandemic" (Group 2) $(\mathrm{n}=24)$ and it was investigated whether there was a significant difference between the parameters. The mean age of the patients in Group 1 was $57.16 \pm 12.30$ years, the mean age of the patients in Group 2 was $55.17 \pm 13.70$ years; no statistically significant difference was observed $(\mathrm{p}=0.575)$. Three $(9.7 \%)$ of the patients in Group 1 were referred to neoadjuvant therapy, compared to $4(16.7 \%)$ in Group 2. Despite the difference between the percentages of patients referred to neoadjuvant therapy, no statistically significant difference was observed $(\mathrm{p}=0.686)$. While the mean tumor size of the patients who underwent surgery in the pre-pandemic period was $3.35 \pm 2.25 \mathrm{~cm}$, it was $3.51 \pm 1.67 \mathrm{~cm}$ in the pandemic period. Although an increase in tumor size was detected between the two groups at the time of diagnosis, this difference was not sufficient to affect staging and was not statistically significant $(\mathrm{p}=0.141)$. Positive SLNB rate was found to be $25.8 \%(n=8)$ in patients in Group 1, while this rate increased to $41.7 \%(\mathrm{n}=10)$ in Group 2, but no statistically significant difference was observed $(\mathrm{p}=0.214)$.

\section{DISCUSSION}

The rapid spread of the SARS-Cov 2 virus has caused the pandemic. Due to the fast human-to-human transmission, many countries continue to impose severe restrictions to limit the spread. These restrictions have changed our daily routines and caused reorganizations in health practices. In addition, non-emergency medical services were suspended during this period. Routine oncology preventive activities also slowed down, especially at the beginning of the pandemic $(3,12)$. In our study, the effect of the pandemic on the clinical staging of breast cancer at the time of diagnosis was investigated.
Although breast cancer is the most common cancer in society and one of the most common causes of cancerrelated deaths in women, recent improvements in prognosis have been observed due to the possibility of early diagnosis in breast cancer and the implementation of screening programs (13-15). One of the factors that most affect survival is the stage of breast cancer (16). It has been reported that the ten-year overall survival and disease-free survival rates of breast cancer patients according to the stages are $67-88 \%$ and $70-75 \%$, respectively (17). Local recurrence rates in these patients are between $8-19 \%$ (18).

However, with the COVID-19 pandemic, screening tests have been interrupted due to the burden on the health system. In the center where the study was conducted, it was observed that the number of outpatient clinic applications and the number of mammographies performed due to breast complaints during the COVID-19 pandemic decreased significantly compared to the previous year. For this reason, clinicians have been concerned about the progression of the breast cancer stage in the population. Unfortunately, there are no extensive studies on this subject in the literature. Tumor size, lymph node positivity, and distant organ metastasis are effective in breast cancer staging (19). Distant organ metastasis was not detected in any of the patients included in the study at the time of the study. For this reason, tumor size and SLNB positivity were examined for comparison in our study.

Despite the suspension of breast cancer screening, we did not observe an increase in $\mathrm{T}$ stages. However, the mean mass size increased from approximately $3.3 \mathrm{~cm}$ to $3.5 \mathrm{~cm}$. The reason for this result may be the low number of patients and the relatively short lockdown time. Although the increase in size is not statistically significant, this increase is essential for the prognosis of the disease (20). Studies are showing that tumor size has a direct effect on survival (21). In addition, studies have shown that tumor size is correlated with positive lymph node numbers $(20,22,23)$. Therefore, Increasing the number of positive lymph nodes and tumor size worsens the prognosis. However, these two clinical outcomes are independent of each other. For example, the prognosis worsens with or without a positive lymph node as the tumor size increases $(20,24)$.

\begin{tabular}{|lcccc|}
\hline Table. & $\begin{array}{c}\text { All Patients } \\
(\mathbf{n}=\mathbf{5 5})\end{array}$ & $\begin{array}{c}\text { Group 1 (before the } \\
\text { pandemic) (n=31) }\end{array}$ & $\begin{array}{c}\text { Group 2 (during } \\
\text { pandemic) (n=24) }\end{array}$ & $\begin{array}{c}\text { Statistical } \\
\text { Significance }\end{array}$ \\
\hline Variables & $56.29 \pm 12.84(58)$ & $57.16 \pm 12.30(59)$ & $55.17 \pm 13.70(56.5)$ & $0.575^{*}$ \\
\hline Age (Years) & $7(\% 12.7)$ & $3(\% 9.7)$ & $4(\% 16.7)$ & $0.686 \dagger$ \\
Neoadjuvant Chemotheraphy & $3.42 \pm 2.00(3)$ & $3.35 \pm 2.25(2.5)$ & $3.51 \pm 1.67(3.05)$ & $0.141^{*}$ \\
Tumor Size (Centimeters) & $1(1-6)$ & $1(1-5)$ & $1.5(1-6)$ & $14(\% 58.3)$ \\
Sentinel Lymph Node Count & $37(\% 67.3)$ & $23(\% 74.2)$ & $10(\% 41.7)$ & $0.380^{*}$ \\
Sentinel Lymph Node Status & Negative & $18(\% 32.7)$ & $8(\% 25.8)$ & \\
\cline { 2 - 6 } & Positive & & &
\end{tabular}


Lymph node metastasis is one of the most important prognostic factors in breast cancer $(20,25)$. When we look at SNLB positivity in our study group, it was observed that it increased from $25,8 \%$ to $41,7 \%$, although it was not statistically significant. This increase is one of the most drastic effects of the pandemic. The study compares two periods of 6 months. Although this period was relatively short, it caused delayed diagnosis in breast cancer patients and also increased both size and lymph node positivity.

Our study contains deficiencies due to the nature of retrospective studies. It has limitations as the lockdown period is relatively short, the number of patients is small, it was performed in a single-center, and some patients did not want to participate in the study. However, it contains valuable information in terms of the fact that it is a study that emphasizes the importance of cancer screening. Breast cancer can be cured if diagnosed in the early stages, and that a critical follow-up process is noted in pandemic conditions other than emergency surgeries. As far as we know, there is no further research on the effects of the pandemic on the stage of breast cancer.

\section{CONCLUSION}

As a result, we believe that breast cancer is one of the diseases that should not be followed up during epidemic periods such as COVID-19, which is rare and affects the whole world, and that society should be informed more. By providing appropriate protection conditions, breast cancer screening and diagnostic evaluations should not be disrupted. Our study showed that even in this short period, there was an increase in tumor sizes and positive lymph node counts. This information is of great importance for more extended lockdown periods.

\section{ETHICAL DECLARATIONS}

Ethics Committee Approval: The study was carried out with the permission of Hitit University NonInterventional Research Ethics Committee (Date: 30.04.2021, Decision No: 2021-66).

Informed Consent: All patients signed the free and informed consent form.

Referee Evaluation Process: Externally peer-reviewed.

Conflict of Interest Statement: The authors have no conflicts of interest to declare.

Financial Disclosure: The authors declared that this study has received no financial support.

Author Contributions: All of the authors declare that they have all participated in the design, execution, and analysis of the paper, and that they have approved the final version.

\section{REFERENCES}

1. Wu Z, McGoogan JM. Characteristics of and important lessons from the coronavirus disease 2019 (COVID-19) outbreak in China: summary of a report of 72314 cases from the Chinese Center for Disease Control and Prevention. JAMA 2020; 323: 1239-42.

2. Organization WH. Novel Coronavirus (2019-nCoV). Situation Report-22, Data as reported by 11 February 2020. 2020.

3. Buonomo OC, Materazzo M, Pellicciaro M, Caspi J, Piccione E, Vanni G. Tor Vergata University-Hospital in the beginning of COVID-19-Era: Experience and recommendation for breast cancer patients. in vivo 2020; 34: 1661-5.

4. Lai AG, Pasea L, Banerjee A, et al. Estimating excess mortality in people with cancer and multimorbidity in the COVID-19 emergency. MedRxiv 2020.

5. Maringe C, Spicer J, Morris M, et al. The impact of the COVID-19 pandemic on cancer deaths due to delays in diagnosis in England, UK: a national, population-based, modelling study. Lancet Oncol 2020; 21: 1023-34

6. İnanç İH, Bursa N, Gültepe A, Şabanoğlu C. The impact of COVID-19 on rural population: A retrospective study. J Health Sci Med 2021; 4: 722-7.

7. Ekiz İșcanlı İG, Şaylan B. Evaluation of factors which determining survival of SARS COV-2 infected patients in intensive care unit. Anatolian Curr Med J 2021; 3: 36-43.

8. Sung H, Ferlay J, Siegel RL, et al. Global cancer statistics 2020: GLOBOCAN estimates of incidence and mortality worldwide for 36 cancers in 185 countries. CA: A Cancer Journal for Clinicians 2021;71: 209-49.

9. Marmot MG, Altman D, Cameron D, Dewar J, Thompson S, Wilcox $\mathrm{M}$. The benefits and harms of breast cancer screening: an independent review. Br J Cancer 2013; 108: 2205-40.

10. Figueroa JD, Gray E, Pashayan N, et al. The impact of the COVID-19 pandemic on breast cancer early detection and screening. Prevent Med 2021; 151: 106585.

11. Peng SM, Yang KC, Chan WP, et al. Impact of the COVID-19 pandemic on a population-based breast cancer screening program. Cancer 2020; 126: 5202-5.

12.Shimazu K, Tamaki Y, Taguchi T, Takamura Y, Noguchi S. Comparison between periareolar and peritumoral injection of radiotracer for sentinel lymph node biopsy in patients with breast cancer. Surgery 2002; 131: 277-86.

13.Pijpers R, Meijer S, Hoekstra OS, et al. Impact of lymphoscintigraphy on sentinel node identification with technetium-99m-colloidal albumin in breast cancer. J Nuclear Med 1997; 38: 366-8.

14. Curigliano G, Cardoso MJ, Poortmans P, et al. Recommendations for triage, prioritization and treatment of breast cancer patients during the COVID-19 pandemic. The Breast 2020;52: 8-16.

15. Buonomo O, Granai A, Felici A, et al. Day-surgical management of ductal carcinoma in situ (DCIS) of the breast using wide local excision with sentinel node biopsy. Tumori J 2002; 88: S48-S9.

16. Dorling L, Carvalho S, Allen J, et al. Breast cancer risk genesassociation analysis in more than 113,000 women. N Engl J Med 2021: 428-39.

17. Cardoso F, Kyriakides S, Ohno S, et al. Early breast cancer: ESMO Clinical Practice Guidelines for diagnosis, treatment and followup. Ann Oncol 2019; 30: 1194-220.

18. Kupstas AR, Hoskin TL, Day CN, Habermann EB, Boughey JC. Effect of surgery type on time to adjuvant chemotherapy and impact of delay on breast cancer survival: a national cancer database analysis. Ann Surg Oncol 2019; 26: 3240-9.

19. Karanlık H, Özmen V, Asoğlu O, et al. Meme kanseri cerrahi tedavisinin uzun dönem sonuçlari. Meme Sağllğ̆ Derg 2006; 2: 2. 
20. Arvold ND, Taghian AG, Niemierko A, et al. Age, breast cancer subtype approximation, and local recurrence after breastconserving therapy. J Clin Oncol 2011; 29: 3885.

21. Veronesi U, Viale G, Rotmensz N, Goldhirsch A. Rethinking TNM: breast cancer TNM classification for treatment decisionmaking and research. The Breast 2006; 15: 3-8.

22. Carter CL, Allen C, Henson DE. Relation of tumor size, lymph node status, and survival in 24,740 breast cancer cases. Cancer 1989; 63: 181-7.

23. Foulkes WD, Reis-Filho JS, Narod SA. Tumor size and survival in breast cancer-a reappraisal. Nat Rev Clin Oncol 2010; 7: 348-53.

24. Fisher B, Slack NH, Bross ID, Investigators C. Cancer of the breast: size of neoplasm and prognosis. Cancer 1969; 24: 1071-80.

25. Olivotto IA, Jackson JS, Mates D, et al. Prediction of axillary lymph node involvement of women with invasive breast carcinoma: a multivariate analysis. Cancer: Interdisciplinary Int J Am Cancer Soc 1998; 83: 948-55.

26. Mook S, Schmidt MK, Rutgers EJ, et al. Calibration and discriminatory accuracy of prognosis calculation for breast cancer with the online Adjuvant! program: a hospital-based retrospective cohort study. Lancet Oncol 2009; 10: 1070-6.

27. Krag DN, Anderson SJ, Julian TB, et al. Sentinel-lymph-node resection compared with conventional axillary-lymph-node dissection in clinically node-negative patients with breast cancer: overall survival findings from the NSABP B-32 randomised phase 3 trial. Lancet Oncol 2010; 11: 927-33. 